\title{
Sudden releases of gases
}

\author{
Hana Chaloupecká ${ }^{1,2, a}$, Zbyněk Jaňour ${ }^{2}$, Klára Jurčáková ${ }^{2}$, Libor Kukačka ${ }^{1,2}$ and Štěpán Nosek ${ }^{2}$ \\ ${ }^{1}$ Charles University in Prague, Faculty of Mathematics and Physics, Department of Meteorology and Environmental \\ Protection, Czech Republic \\ ${ }^{2}$ Institute of Thermomechanics Academy of Sciences of the Czech Republic, v.v.i. Dolejškova 1402/5, Prague 182 00, \\ Czech Republic
}

\begin{abstract}
Conurbations all over the world have enlarged for numberless years. The accidental or intentional releases of gases become more frequent. Therefore, these crises situations have to be studied. The aim of this paper is to describe experiments examining these processes that were carried out in the laboratory of Environmental Aerodynamics of the Institute of Thermomechanics AS CR in Nový Knín. Results show huge puff variability from replica to replica.
\end{abstract}

\section{Introduction}

Air pollution affects human incessantly everywhere. It can be smoke from factory stacks, gases from car exhausts, for instance. But there are also gases that can be dangerous if they are released to the atmosphere - for example combat gases, chlorine, ammonia. Some of them are presented in every town - chlorine is used in swimming pools, ammonia in cooling chambers or winter stadiums. Therefore, these crises situations have to be studied. The most danger of gas leakage of hazardous gases is during their transport. Truck can crash and there may be formed a leak in cistern. Gas leakage duration is rather short.

Studies engaged in dispersion of pollution are mostly concentrated on continuous gas sources. Mathematic models cope with plume-like dispersion behaviour better than puff-like dispersion behaviour. Research dealing with sudden releases of gases is described for example in [1], [2] or [3].

In this paper we will focus our attention on passive short duration gas discharges in idealized housing development typical for inner parts of cities in Europe. We will define puff characteristics. And moreover, we will look at the influence of gas release duration on them.

\section{Experimental set-up}

\subsection{Wind tunnel}

The experiments examining processes of sudden releases of gases were carried out in the Laboratory of Environmental Aerodynamics of the Institute of Thermomechanics AS CR in Nový Knín. The scheme of

\footnotetext{
${ }^{\mathrm{a}}$ hana.chaloupecka@it.cas.cz
}

low speed wind tunnel is depicted in figure 1 . The test section of the tunnel is $2 \mathrm{~m}$ long; its cross-dimensions are $1.5 \mathrm{~m} \times 1.5 \mathrm{~m}$. The wind tunnel parameters are more detailed described in [4].

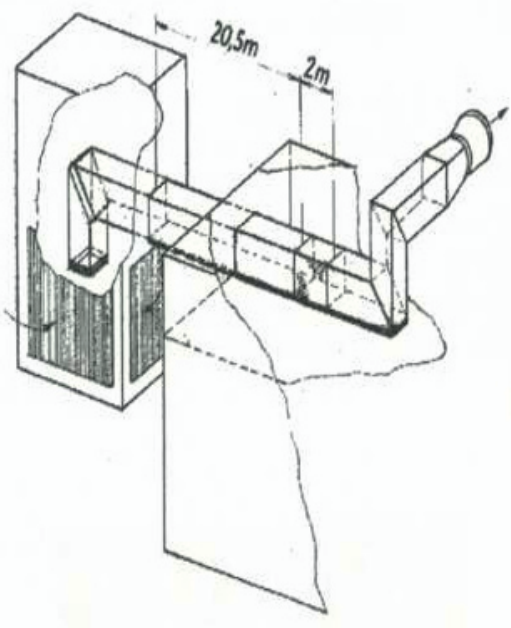

Figure 1. Scheme of open low speed wind tunnel.

The fully turbulent boundary layer was developed by placement of spires and roughness elements in the $20.5 \mathrm{~m}$ long development section (see figure 2). 


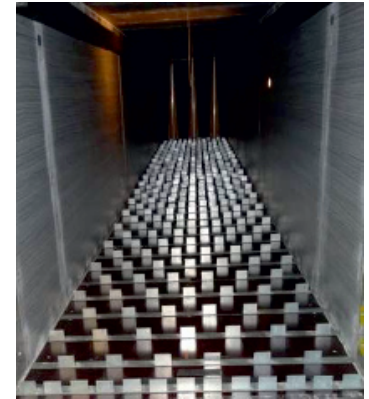

Figure 2. Boundary layer development in the wind tunnel.

\subsection{Model}

The model of idealized urban area is displayed in figure 3. Model manufacturing scale is $1: 400$. Buildings are $63 \mathrm{~mm}$ height $(50 \mathrm{~mm}$ body of buildings with $13 \mathrm{~mm}$ pitched roofs). The buildings width is $37.5 \mathrm{~mm}$. These buildings are formed into closed yards with dimensions $150 \mathrm{~mm} \times 300 \mathrm{~mm}$. Yards were placed $50 \mathrm{~mm}$ from each other (see figure 4 ).

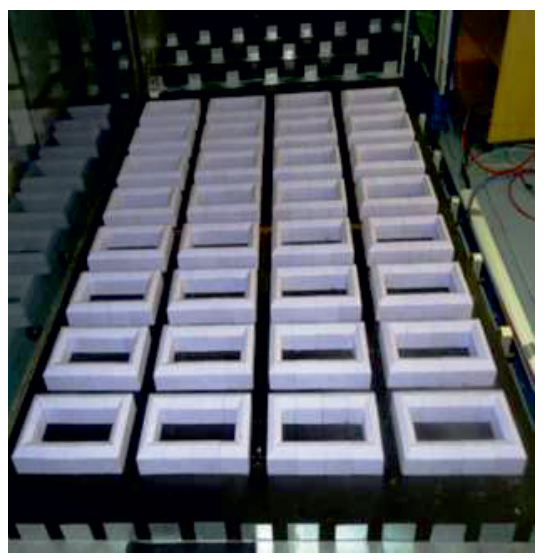

Figure 3. Model of idealized urban area.

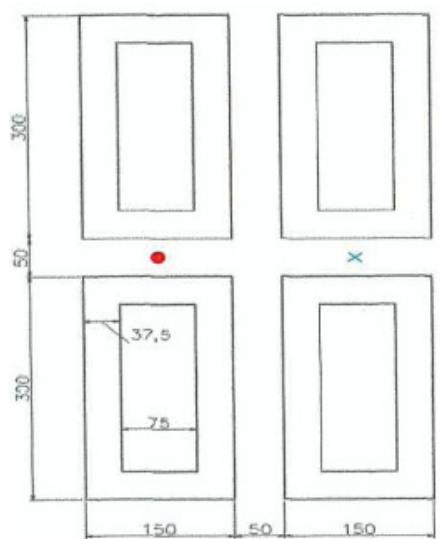

Figure 4. Scheme of meted section with dimensions.

\subsection{Simulations of sudden releases of gases}

In our experiments we used a point ground level gas source. It was placed in the position highlighted by a dot in figure 4. Moreover, you can see a cross symbol representing the detector placement. We used FastResponse-Flame-Ionisation-Detector HFR400 of Cambustion Ltd. for concentration measurements. As a trace gas we used ethan.

Short-time duration discharge was produced by Programmable Logic Controller (PLC) Siemens LOGO! 12/24 RCE 0BA 7 and electromagnetic $3 / 2$ valve ND5 (see figure 5).

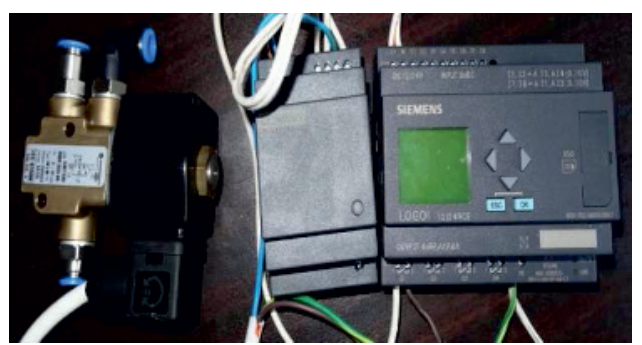

Figure 5. Equipment for puff simulations.

Puffs were created according to the following procedure:

The gas is transported in a hose to input valve orifice.

a) It goes continually through the output orifice in figure 6 the right upper - until the voltage is set to the valve by PLC.

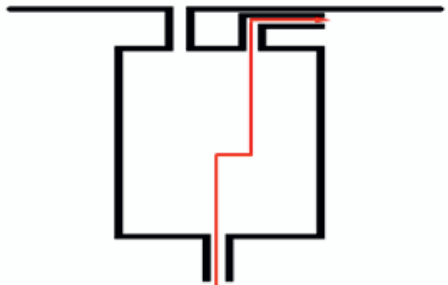

Figure 6. Puff simulations - valve without voltage.

b) While the valve is under voltage, gas is transported to the second output orifice - in figure 7 the left upper orifice - that empties into the bottom of the tunnel and "cloud" of gas is created in the wind tunnel.

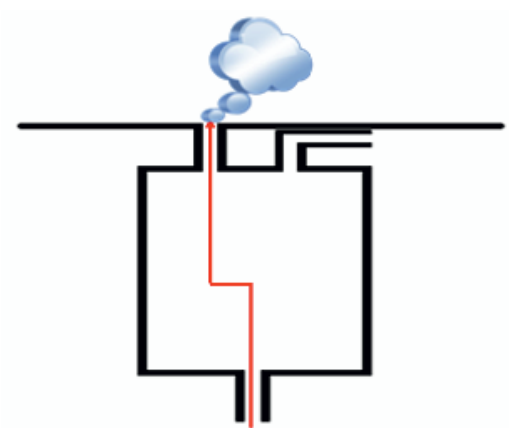

Figure 7. Puff creation - valve under voltage. 


\section{Physical quantities}

In this article we will use dimensionless quantities. Diagrams will have dimensionless time $\mathrm{t}^{*}=\mathrm{tU}_{\text {ref }} / \mathrm{L}_{0}$ on horizontal axis, while vertical axis will displays dimensionless concentration $\mathrm{C}^{*}=\mathrm{CU}_{\mathrm{ref}} \mathrm{L}_{0}{ }^{2} / \mathrm{Q}$. In these relations $\mathrm{U}_{\text {ref }}$ stands for reference speed, $\mathrm{L}_{0}$ characteristic length (in this experiment buildings height), Q source intensity.

\section{Puff description}

Independently the same environmental conditions, two following puffs will never be the same (see figure 8). While in figure 8 (a) the puff "cloud" is split into more parts that is not true for the puff in figure $8(\mathrm{~b})$. The reason for this strong variability in puff appearance is turbulent wind that influence puff transport and dispersion. Therefore many experiments (several hundred) under the same release conditions in the wind tunnel have to be carried out. Only by this procedure we can obtain statistically representative results.

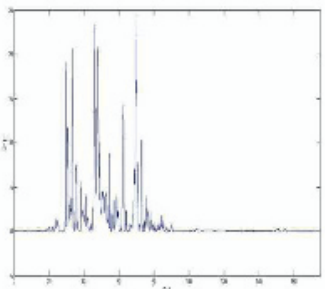

(a)

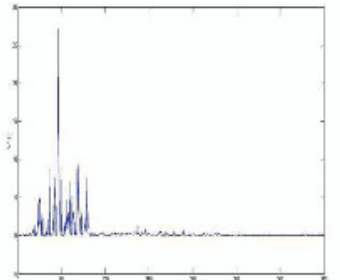

(b)
Figure 8. Comparison of 2 replicas.

If we have an ensemble of sudden releases of gas conducted under the same release conditions large enough, we can also calculate a mean puff from the data. However, this figure won't show us puff variability.

To analyse puff diversity there have to be defined some puff characteristics. In this article we will focus our attention on the following ones (see also figure 9), the definition of which is based on [5]:

\subsection{Arrival time}

Arrival time (AT*) is the time when a gas "cloud" attaches the point where we measure for the first time. In this article we will use the definition based on threshold if measured concentration exceed this value and stay above it for a specified time period we will say that the puff "cloud" has appeared in the place.

\subsection{Peak concentration}

Peak concentration $\left(\mathrm{PC}^{*}\right)$ is the maximum concentration recorded during measurement.

\subsection{Peak time}

Peak time $\left(\mathrm{PT}^{*}\right)$ is the time when maximum concentration was detected.

\subsection{Ascent time period}

Ascent time period (ASCT*) is the time period between arrival and peak time.

\subsection{Leaving time}

Leaving time (LT*) is the time when a puff "cloud" leaves the point of measurement. Like for arrival time we will use here the definition based on threshold - when concentration decrease under the threshold and remains bellow it till the next gas discharge, we will say that the gas "cloud" definitely left the measurement point.

\subsection{Descent time period}

Descent time period (DSCT ${ }^{*}$ ) is the time period between peak and leaving time.

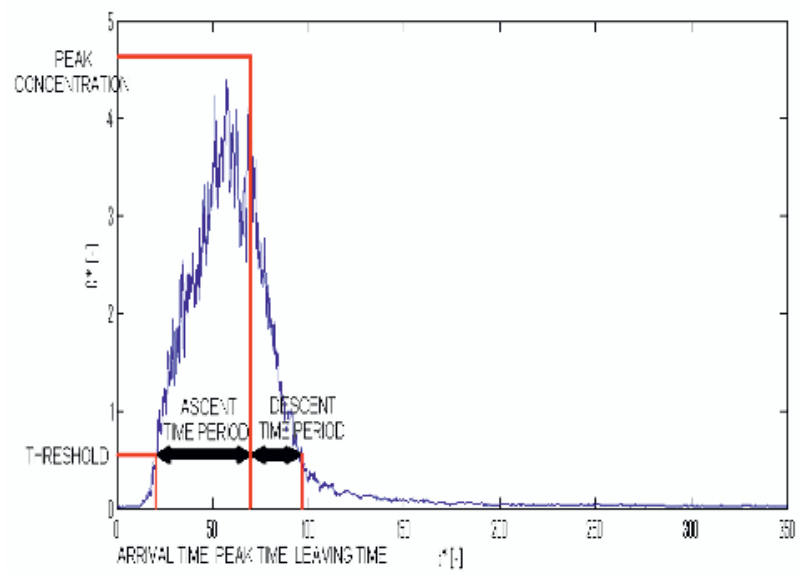

Figure 9. Puff characteristics

\section{Results}

We will discuss here results for puffs with experimental release times $2.5 \mathrm{~s}$ and $5 \mathrm{~s}$. In figure 10 there are depicted mean puffs (from all replicas) for both release times. Characteristics of these mean puffs are shown in Table 1.

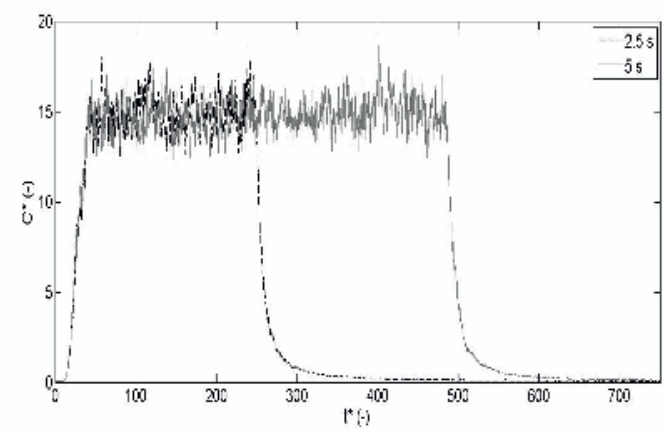

Figure10. Comparison of $2.5 \mathrm{~s}$ and $5 \mathrm{~s}$ mean puffs. 
Table 1. Characteristics of mean puffs.

\begin{tabular}{|c|c|c|}
\hline release time [s] & $\mathbf{2 . 5}$ & $\mathbf{5}$ \\
\hline AT* (-) $^{*}$ & 23 & 23 \\
PC* (-) $^{*}$ & 19 & 19 \\
PT* (-) $^{*}$ & 241 & 402 \\
ASCT $^{*}(-)$ & 218 & 379 \\
LT* (-) $^{*}$ & 259 & 498 \\
DSCT $^{*}(-)$ & 17 & 97 \\
\hline
\end{tabular}

Variance of time when a gas cloud attaches the point where concentration is measured is mostly caused by large eddies motions. They cause meander of the cloud. In figure 11 (a) and (b) there are depicted frequency distributions for dimensionless arrival times for $2.5 \mathrm{~s}$ and $5 \mathrm{~s}$ release times. Arrival times don't much differ, if we compare differences in mean puffs (Table 1), arrival times are the same value for $2.5 \mathrm{~s}$ and $5 \mathrm{~s}$ release time.

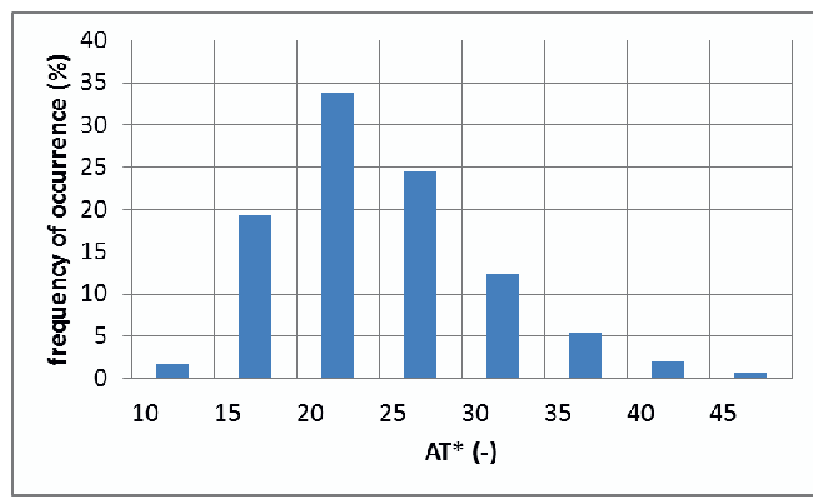

(a) release time $2.5 \mathrm{~s}$

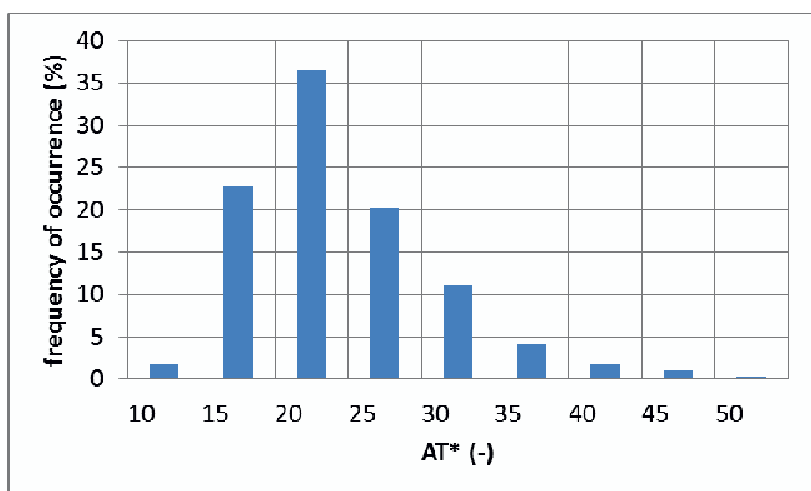

(b) release time $5 \mathrm{~s}$

Figure 11. Arrival time.

On the contrary eddies of a similar and smaller scale like the puffs cause cloud material redistribution, shape of the cloud is distort, puff can be even split in more parts. Therefore peak concentration varies from replica to replica. Peak concentration of $2.5 \mathrm{~s}$ and $5 \mathrm{~s}$ mean puffs are the same as shown in Table 1. More detailed comparison (figure 12 (a) and (b)) shows that there is higher frequency of maximum concentration occurrence at lower values at $2.5 \mathrm{~s}$ puffs than at $5 \mathrm{~s}$. $5 \mathrm{~s}$ puffs contrariwise have larger peak values at huger values than $2.5 \mathrm{~s}$ puffs. The reason is that maximum concentration can be detected whenever during the time gas cloud is presented at sampler place (see figure 13 (a), (b)). When time release grows in size form $2.5 \mathrm{~s}$ to $5 \mathrm{~s}$, time during which bigger maximum concentration can occur is longer.

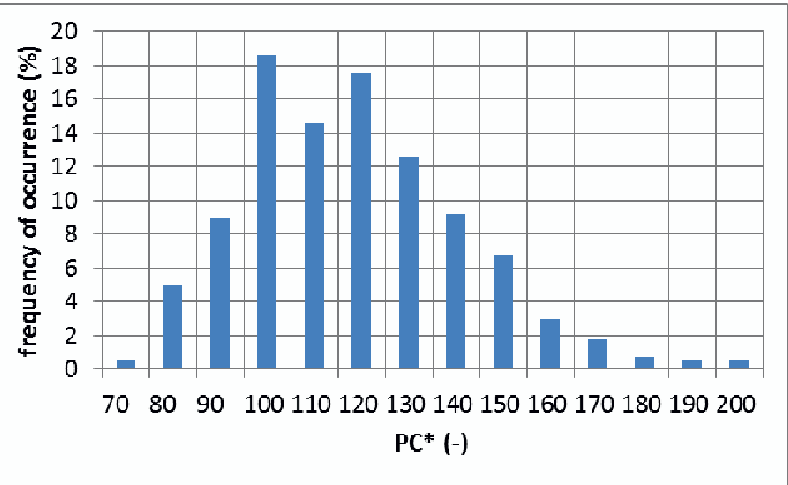

(a) release time $2.5 \mathrm{~s}$

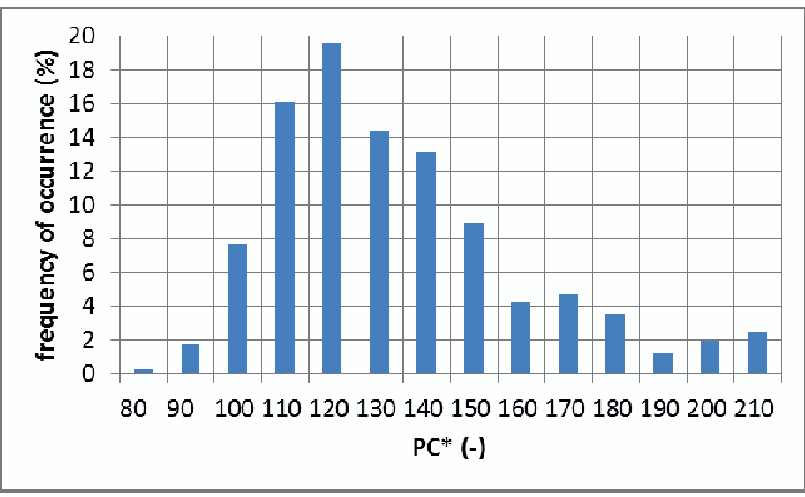

(b) release time $5 \mathrm{~s}$

Figure 12. Peak concentration.

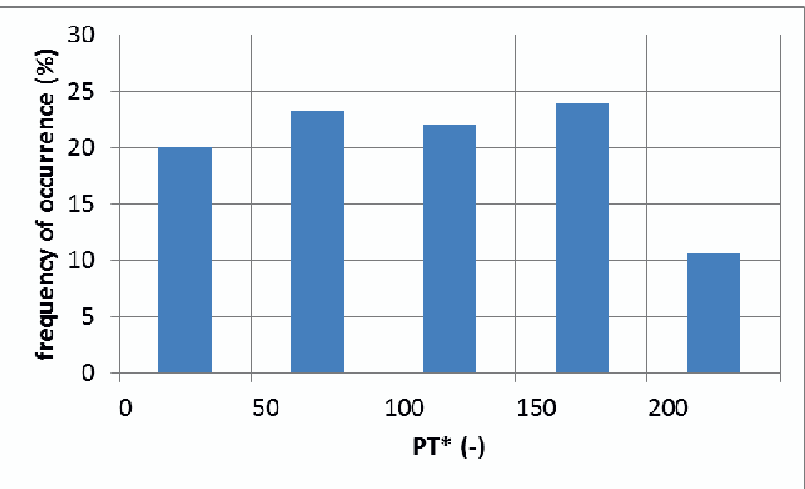

(a) release time $2.5 \mathrm{~s}$

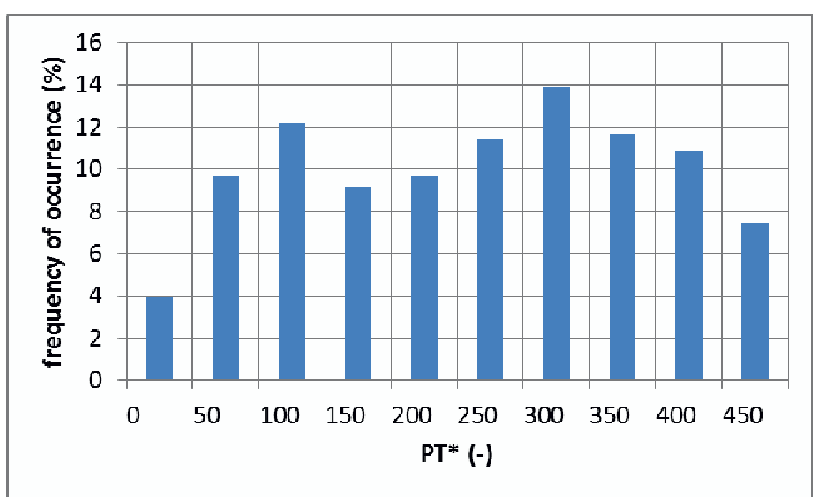

(b) release time $5 \mathrm{~s}$

Figure 13. Peak time. 
Since time when maximum concentration is measured may be whichever time of gas cloud presence at sampler point, ascent time period varies a lot (see figure 14 (a), (b)).

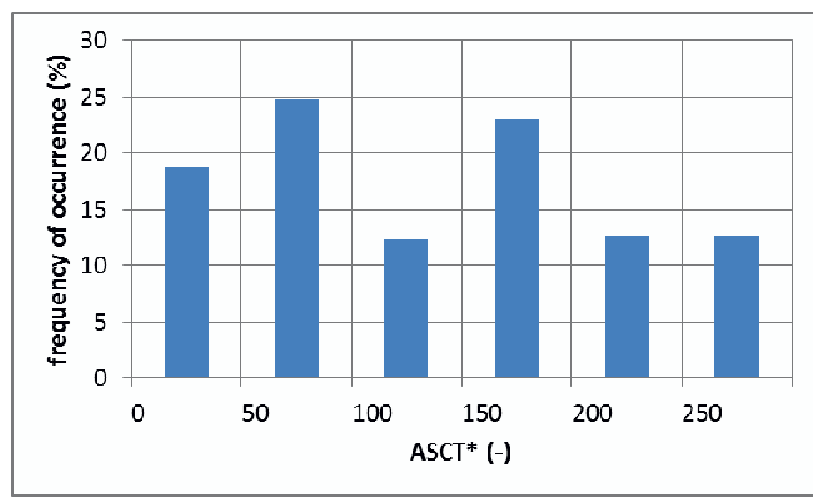

(a) release time $2.5 \mathrm{~s}$

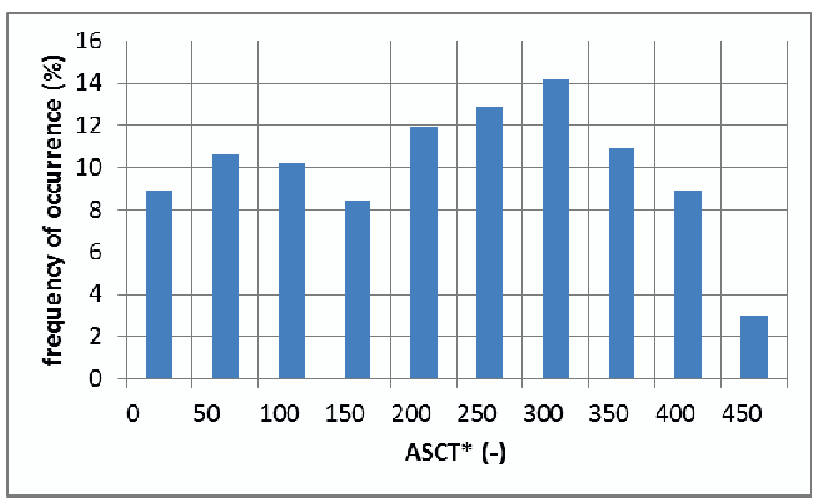

(b) release time $5 \mathrm{~s}$

Figure 14. Ascent time period.

Eddies are also one of reasons why leaving time (see figure 15 (a), (b)) varies from replica to replica. Moreover, variance is caused also by the leaving time definition itself - based on a threshold. Too high threshold causes cutting away of puff edge, too small missinterpretation of background concentration as a gas cloud. Comparison of 2.5 and $5 \mathrm{~s}$ mean puffs shows that $5 \mathrm{~s}$ mean leaving time is a little shorter than double leaving time of $2.5 \mathrm{~s}$ mean leaving time. If we have a look at diagram of dimensionless leaving time frequency distributions for release time $2.5 \mathrm{~s}$, we will see that maximum percentage of occurrence is in interval between 270 and 280 . More than twofold value of 270 has only less than $5 \%$ of puffs at $5 \mathrm{~s}$ mean puff.

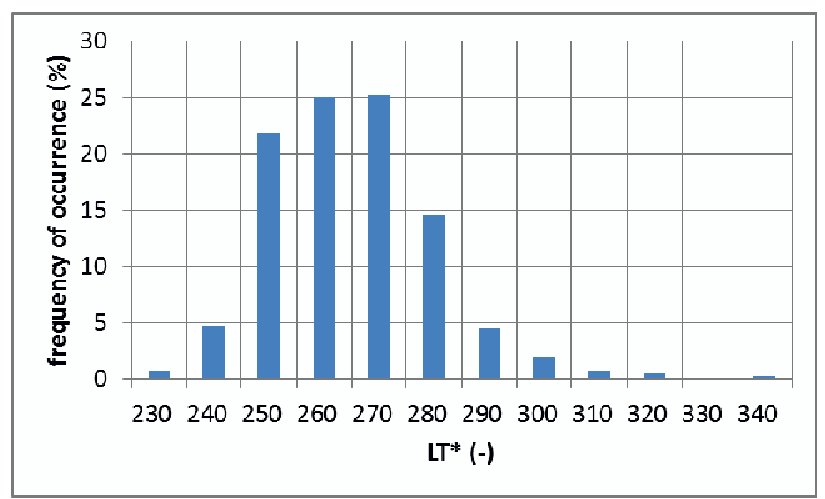

(a) release time $2.5 \mathrm{~s}$

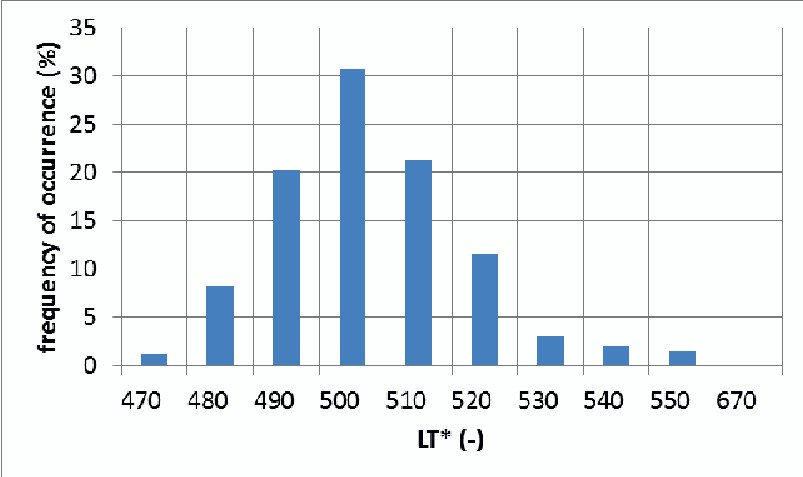

(b) release time $5 \mathrm{~s}$

Figure 15. Leaving time.

Like ascent time period also descent time period varies a lot for the same reason (see figure 16 (a), (b)).

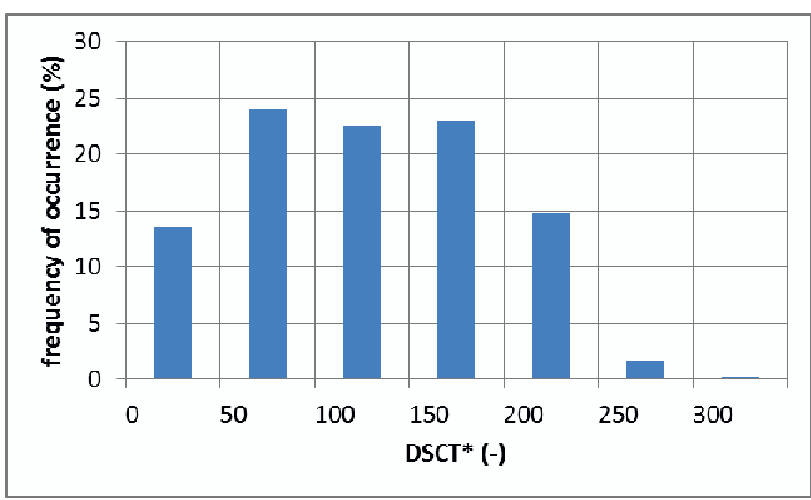

(a) release time $2.5 \mathrm{~s}$

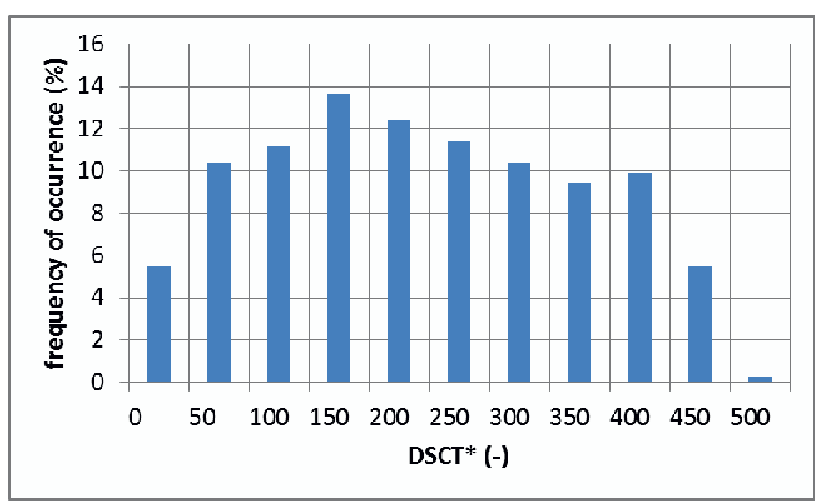

(b) release time $5 \mathrm{~s}$

Figure 16. Descent time period.

\section{Conclusions}

Sudden releases of gases (short duration dangerous gas discharge during a terrorist attack or an accident) are greatly different from leaks form continuous gas sources (smoke from factory stacks, car exhausts). While for studying continuous gas sources corresponding experiment in wind tunnel suffices to be conducted only once for concentration calculation there must be carried out many replicas under the same release conditions to be able to evaluate puffs concentration.

Comparison of passive gas discharges of duration $5 \mathrm{~s}$ and $2.5 \mathrm{~s}$ shows that the time when a gas cloud reaches the sampler point doesn't much differ. $5 \mathrm{~s}$ puffs have 
higher frequency of maximum concentration occurrence at larger values than $2.5 \mathrm{~s}$ puffs. Moreover, they have lower frequency of leaving time occurrence at larger values in comparison with twofold leaving time value of puffs with $2.5 \mathrm{~s}$ release time. Length of ascent and descent time periods vary a lot in both $-2.5 \mathrm{~s}$ and $5 \mathrm{~s}$ puffs.

\section{Acknowledgements}

Authors thank for kind support from LD12007 and GAČR GAP101/12/1554.

\section{References}

1. P.C. Chatwin. The dispersion of a puff of passive contaminant in the constant stress region. Q J Royal Met. Soc., 94, 350-360 (1968)

2. R.N. Meroney, A. Lohmeyer. Statistical characteristics of instataneous dense gas clouds released in an atmospheric boundary-layer wind tunnel. Boundary-Layer Meteorology, 28, 1-22 (1984)

3. A.G. Robins, J.E. Fackrell. An experimental study of the dispersion of short duration emissions in a turbulent boundary layer. Proc. Air Pollution VI, WIT Press (1998)

4. Z. Jaňour. Modelování mezní vrstvy atmosféry (Karolinum, 2001)

5. R. Fisher, M. Schatzmann, B. Leitl. How often is sufficient? A program for the statistical analysis of puff dispersion in urban environments. Proc. Physmod (2007) 